\title{
Asymptotic symmetries of three dimensional gravity and the membrane paradigm
}

\author{
Mariana Carrillo-González ${ }^{a}$ and Robert F. Penna ${ }^{b}$ \\ ${ }^{a}$ Center for Particle Cosmology, Department of Physics and Astronomy, \\ University of Pennsylvania, \\ 209 S. 33rd St., Philadelphia, PA 19104, U.S.A. \\ ${ }^{b}$ Center for Theoretical Physics, Department of Physics, Columbia University, \\ New York, NY 10027, U.S.A. \\ E-mail: cmariana@sas.upenn.edu, rp2835@columbia.edu
}

ABSTRACT: The asymptotic symmetry group of three-dimensional (anti) de Sitter space with Brown-Henneaux boundary conditions is the two dimensional conformal group with central charge $c=3 \ell / 2 G$. Usually the asymptotic charge algebra is derived using the symplectic structure of the bulk Einstein equations. Here, we derive the asymptotic charge algebra by a different route. First, we formulate the dynamics of the boundary as a 1+1dimensional dynamical system. Then we realize the boundary equations of motion as a Hamiltonian system on the dual Lie algebra, $\mathfrak{g}^{*}$, of the two-dimensional conformal group. Finally, we use the Lie-Poisson bracket on $\mathfrak{g}^{*}$ to compute the asymptotic charge algebra. This streamlines the derivation of the asymptotic charge algebra because the Lie-Poisson bracket on the boundary is significantly simpler than the symplectic structure derived from the bulk Einstein equations. It also clarifies the analogy between the infinite dimensional symmetries of gravity and fluid dynamics.

KEywords: Classical Theories of Gravity, Space-Time Symmetries

ArXiv EPrint: 1810.06954 


\section{Contents}

1 Introduction 1

2 Virasoro algebra $\quad 2$

3 Anti-de Sitter 5

3.1 Boundary dynamics 6

3.2 Charge algebra 8

3.3 Membrane paradigm 8

4 de Sitter $\quad 10$

$\begin{array}{ll}4.1 \text { Conserved quantities } & 11\end{array}$

$\begin{array}{lll}4.2 & \text { Example: Kerr-dS } & 12\end{array}$

5 Discussion $\quad 12$

\section{Introduction}

The space of solutions of the Einstein equations has a huge gauge symmetry: metrics differing by small diffeomorphisms ${ }^{1}$ are physically equivalent. This introduces a great deal of complexity into the construction of a symplectic structure on this space $[1,2]$. However, when the phase space of a dynamical system carries redundant information, it is often possible to "quotient out" the redundancy through the process of symplectic reduction and obtain a simpler description of the same dynamics. An extremely simple example appears in rigid body dynamics. In this case, the configuration space is the group $G=\mathrm{SO}(3)$ and the canonical phase space is the cotangent bundle, $T^{*} G$. However, the Euler equations of rigid body dynamics are invariant under the left action of $G$ on $T^{*} G \cong G \times \mathfrak{g}^{*}$, so we can quotient out by $G$ and obtain an equivalent Hamiltonian system on $\mathfrak{g}^{*}$, the dual of the Lie algebra. ${ }^{2}$ In this process, the canonical Poisson bracket on $T^{*} G$ descends to the Lie-Poisson bracket on $\mathfrak{g}^{*}$ and the dimension of phase space is reduced by half [3].

In this paper, we consider similar examples in general relativity. Our examples are three dimensional gravity with asymptotically de Sitter (dS) and anti de Sitter (AdS) BrownHenneaux boundary conditions [4] (variations of Brown-Henneaux boundary conditions are possible, see e.g., [5-8]). In either case, the space of physically distinct solutions of the Einstein equations is parametrized by boundary data supported at asymptotic infinity. The projection of the Einstein equations onto asymptotic infinity gives a set of constraints on the boundary data. We regard the constraints as equations of motion for a dynamical

\footnotetext{
${ }^{1}$ Here "small diffeomorphisms" means diffeomorphisms which act trivially at asymptotic infinity.

${ }^{2}$ Of course, in this example the redundancy is not a gauge redundancy.
} 
system supported on the 1+1-dimensional boundary. This viewpoint is closely related to the black hole membrane paradigm, which treats the projection of Einstein's equation onto a black hole event horizon as a lower dimensional dynamical system [9-11]. Physically, the dynamics is equivalent to conservation of the boundary's Brown-York stress tensor.

We show that the dynamics so obtained can be regarded as Hamiltonian flow on $\mathfrak{g}^{*}$, where now $G$ is the asymptotic symmetry group of the family of spacetimes under consideration and $\mathfrak{g}^{*}$ is the dual of its Lie algebra. In both of our examples, $G$ is two copies of the Virasoro group with central charge fixed by the boundary equations of motion and the normalization of the Brown-York stress energy tensor. As in the rigid body example, the Poisson bracket is the Lie-Poisson bracket and the simplicity of the Lie-Poisson bracket streamlines the computations. The resulting formalism resembles the Hamiltonian description of the compressible Euler equations [12], and we use this analogy to clarify the relationship between conservation laws in fluid dynamics and gravity (see also [13-16]). We note that the Lie-Poisson bracket has appeared in earlier studies of AdS gravity [17-19] but the relationship we draw to the membrane paradigm and fluid dynamics is new. Recently, a new family of boundary conditions has been considered for three dimensional AdS gravity which leads to a set of Korteweg-de Vries (KdV) equations governing the boundary degrees of freedom $[7,8]$. This gives a relationship between gravity and fluid dynamics that is similar in spirit to the present work.

The examples in this paper are closely related to the description of three dimensional asymptotically flat gravity in [20]. In the future, it would be interesting to derive the Lie-Poisson brackets appearing in all these examples from the Poisson bracket on covariant phase space $[1,2]$ via symplectic reduction. In the meantime, the subject of asymptotic symmetries remains of great current interest for its wide array of applications, ranging from consistency relations in cosmology [21-23] to soft theorems in quantum field theory and memory effects in gravitational wave experiments (for a recent review see [24]). The perspective we develop here can, in some cases, allow one to identify asymptotic symmetry groups and compute their charge algebras with relative ease, tools which should prove useful in this rapidly expanding subject.

\section{Virasoro algebra}

In this section, we collect some standard facts about the Virasoro algebra (see, e.g., [3, 25]) that will be needed in the following sections. This establishes notation and keeps the present paper self-contained.

The Virasoro group is a central extension of Diff $\left(S^{1}\right)$, the diffeomorphism group of the circle. So we start by describing $\operatorname{Diff}\left(S^{1}\right)$. The elements of $\operatorname{Diff}\left(S^{1}\right)$ are smooth invertible maps $S^{1} \rightarrow S^{1}$, the group multiplication is composition, and the Lie algebra is $\operatorname{Vect}\left(S^{1}\right)$, the algebra of vector fields on the circle. The Lie bracket is the usual vector field commutator. As a vector space, $\operatorname{Vect}\left(S^{1}\right)$ has a dual space, the space of linear functionals on $\operatorname{Vect}\left(S^{1}\right)$. It is convenient to identify the dual space of $\operatorname{Vect}\left(S^{1}\right)$ with the space of quadratic differentials on $S^{1}$. Given a quadratic differential, $u(\theta) d \theta^{2}$, and a vector field, 
$f(\theta) \partial_{\theta}$, we form the pairing,

$$
\left\langle f(\theta) \partial_{\theta}, u(\theta) d \theta^{2}\right\rangle=\int_{S^{1}} f(\theta) u(\theta) d \theta .
$$

For each quadratic differential, this pairing defines a linear map $\operatorname{Vect}\left(S^{1}\right) \rightarrow \mathbb{R}$. So the dual of $\operatorname{Vect}\left(S^{1}\right)$ becomes identified with the space of quadratic differentials. As vector spaces, $\operatorname{Vect}\left(S^{1}\right)$ and its dual are isomorphic. However, vector fields and quadratic differentials transform differently under diffeomorphisms. This will be important in what follows.

The Virasoro algebra is a central extension of $\operatorname{Vect}\left(S^{1}\right)$. As a vector space it is $\mathfrak{v i r}=$ $\operatorname{Vect}\left(S^{1}\right) \times i \mathbb{R}$ (the factor of $i$ and similar factors below are conventional and simplify some formulas). So an element of $\mathfrak{v i r}$ is a pair, $\left(f(\theta) \partial_{\theta},-i a\right)$, where $a \in \mathbb{R}$. Elements of vir are added and subtracted in the obvious way. What is nontrivial is the Lie bracket on vir. It has the form

$$
\left[\left(f(\theta) \partial_{\theta},-i a\right),\left(b(\theta) \partial_{\theta},-i b\right)\right]=\left(\left[f(\theta) \partial_{\theta}, g(\theta) \partial_{\theta}\right], \frac{-i}{48 \pi} \omega\left(f(\theta) \partial_{\theta}, g(\theta) \partial_{\theta}\right)\right) .
$$

The first entry on the r.h.s. is the usual vector field commutator. The function appearing in the second entry, $\omega\left(f(\theta) \partial_{\theta}, g(\theta) \partial_{\theta}\right)$, is a bilinear function of vector fields. The form of $\omega$ is constrained by the requirement that the bracket (2.2) is indeed a Lie bracket, and in particular that it satisfies the Jacobi identity. This requirement is so severe that there is in fact a unique solution (up to rescalings),

$$
\omega\left(f(\theta) \partial_{\theta}, g(\theta) \partial_{\theta}\right)=2 \int_{S^{1}} f^{\prime}(\theta) g^{\prime \prime}(\theta) \mathrm{d} \theta
$$

where $^{\prime} \equiv \mathrm{d} / \mathrm{d} \theta$. Equations (2.2) and (2.3) define the Lie bracket on the Virasoro algebra.

At this point it is helpful to introduce a basis for vir and express the Virasoro commutation relations in their usual form. The usual basis elements $(m \in \mathbb{Z})$ are

$$
v_{m}=\left(e^{i m \theta} \partial_{\theta},-\frac{i}{24} \delta_{m}^{0}\right)
$$

Any element of $\mathfrak{v i r}$ can be expressed as a linear combination of $v_{m}$. The second entry on the rhs, $-i / 24 \delta_{m}^{0}$, is conventional and we will comment on its significance in a moment. For now, observe that plugging the basis elements into the Lie bracket gives

$$
i\left[v_{m}, v_{n}\right]=(m-n) v_{m+n}+\frac{1}{12}\left(m^{3}-m\right) \delta_{m+n}^{0} \mathcal{Z}
$$

where $\mathcal{Z} \equiv(0,-i)$. These commutation relations are often presented as the definition of the Virasoro algebra. The advantage of starting from the basis-independent presentation (2.2)(2.3) is that we are free to switch to bases other than the $v_{m}$. This will become useful below.

The central extension appears in (2.5) as a term proportional to $\mathcal{Z}$, with contributions linear and cubic in $m$. The linear in $m$ contribution could have been eliminated by setting the second entry of $v_{m}$ to zero in (2.4). However, it is standard to include this term. It has the convenient effect of making the central term vanish for commutators involving only $v_{0}$ 
and $v_{ \pm 1}$. This will not be important for us, but we follow convention. Note that the $m^{3}$ term cannot be eliminated by changing the basis elements. Non-trivial central extensions are classified by 2-cocycles on $\mathfrak{v i r}$, which are elements of the second cohomology $H^{2}(\mathfrak{v i r}, \mathbb{R})$. This is one-dimensional and generated by $\omega\left(f(\theta) \partial_{\theta}, g(\theta) \partial_{\theta}\right)$ [25]. The term linear in $m$ is a 2-coboundary and can be removed by performing a change of basis.

Returning to the general theory, consider the dual space, $\mathfrak{v i r}^{*}$, the space of linear functionals on vir. Its elements are pairs, $\left(u(\theta) d \theta^{2}, i c\right)$. The pairing between $\mathfrak{v i r}$ and $\mathfrak{v i r}^{*}$ is

$$
\left\langle\left(f(\theta) \partial_{\theta},-i a\right),\left(u(\theta) d \theta^{2}, i c\right)\right\rangle=\int_{S^{1}} f(\theta) u(\theta) d \theta+a c .
$$

For each element of $\mathfrak{v i r}^{*}$, this pairing gives a linear map $\mathfrak{v i r} \rightarrow \mathbb{R}$. This justifies our identification of $\mathfrak{v i r}^{*}$ with the dual space of $\mathfrak{v i r}$. As before, note that $\mathfrak{v i r}$ and $\mathfrak{v i r}^{*}$ are isomorphic as vector spaces but they transform differently under diffeomorphisms. Elements of vir are called adjoint vectors and elements of $\mathfrak{v i r}^{*}$ are called coadjoint vectors. The adjoint action of $\mathfrak{v i r}$ on itself is just the Lie bracket (2.2):

$$
\operatorname{ad}_{\left(f(\theta) \partial_{\theta},-i a\right)}\left(g(\theta) \partial_{\theta},-i b\right)=\left(\left[f(\theta) \partial_{\theta}, g(\theta) \partial_{\theta}\right], \frac{-i}{48 \pi} \omega\left(f(\theta) \partial_{\theta}, g(\theta) \partial_{\theta}\right)\right) .
$$

Note that $a$ and $b$ do not enter the r.h.s. . This reflects the fact that the extension is central, i.e., that $\mathbb{R}$ is in the center of vir. To streamline notation we will sometimes not write $a$ and $b$ explicitly on the l.h.s. either.

The adjoint action can be transported to $\mathfrak{v i r}^{*}$ using the pairing (2.6). This defines the coadjoint action,

$$
\operatorname{ad}_{\left(f(\theta) \partial_{\theta},-i a\right)}^{*}\left(u(\theta) d \theta^{2}, i c\right)=-\left(\left(u^{\prime} f+2 u f^{\prime}-\frac{c}{24 \pi} f^{\prime \prime \prime}\right) d \theta^{2}, 0\right) .
$$

The first thing to note is that the coadjoint action is different from the adjoint action (2.7). This is why the distinction between $\mathfrak{v i r}$ and $\mathfrak{v i r} \mathfrak{r}^{*}$ is important: adjoint vectors and coadjoint vectors transform differently under infinitesimal diffeomorphisms (an infinitesimal diffeomorphism is a vector field). In physical problems that arise "in the wild," it is not always obvious at first glance that a field is an element of $\mathfrak{v i r}^{*}$. The way to check this is to see if the field transforms under diffeomorphisms according to the Virasoro coadjoint action (2.8). In problems with hidden Virasoro symmetry, the third derivative in (2.8) often manifests as a signal of the underlying symmetry.

The infinitesimal coadjoint action (2.8) can be integrated to get an action of $\operatorname{Diff}\left(S^{1}\right)$ on $\mathfrak{v i r}^{*}$. The result is

$$
\operatorname{Ad}_{\eta^{-1}}^{*}\left(u(\theta) d \theta^{2}, i c\right)=\left(u(\eta) \cdot\left(\eta^{\prime}\right)^{2} d \theta^{2}-\frac{c}{24 \pi} \operatorname{Sch}(\eta) d \theta^{2}, i c\right)
$$

where $\eta \in \operatorname{Diff}\left(S^{1}\right)$ and $\operatorname{Sch}(\eta)=\left(\eta^{\prime} \eta^{\prime \prime \prime}-\frac{3}{2}\left(\eta^{\prime \prime}\right)^{2}\right) /\left(\eta^{\prime}\right)^{2}$ is its Schwarzian derivative. Note that the central charge, $c$, is invariant under the coadjoint action. In other words, the central charge is constant on orbits of the coadjoint action. Coadjoint orbits are symplectic manifolds and can serve as classical phase spaces. Quantizing a coadjoint orbit gives an irreducible representation of the group [26]. When we speak of "the" central charge of 
a classical physical system, we mean the phase space is a (possibly infinite) product of Virasoro coadjoint orbits with central charge $c$. In the case of three dimensional gravity, a superselection rule forbids superpositions of states with different central charges [27]. This is similar to the superselection rule forbidding superpositions of states with different masses in a system with Galilean symmetry [28].

The dual of the Lie algebra, vir $^{*}$, has a Poisson bracket called the Lie-Poisson bracket. A Poisson bracket is an antisymmetric bilinear map satisfying Leibniz's rule and the Jacobi identity. Let $F$ and $G$ be functions on $\mathfrak{v i r}^{*}$. At a fixed point $\mu \equiv\left(u(\theta) d \theta^{2}, i c\right) \in \mathfrak{v i r}^{*}$, the Lie-Poisson bracket is

$$
\{F, G\}(\mu)=\left\langle\mu,\left[\frac{\delta F}{\delta \mu}, \frac{\delta G}{\delta \mu}\right]\right\rangle .
$$

On the rhs, $\delta F / \delta \mu$ and $\delta G / \delta \mu$ are functional derivatives, considered as elements of vir, and the bracket is the Lie bracket. Equipped with the Lie-Poisson bracket, vir* can serve as a phase space for Hamiltonian systems. Let $H$ be a function on $\mathfrak{v i r}^{*}$, then Hamilton's equations are given by

$$
\frac{\partial F}{\partial t}=\{F, H\}
$$

Expand the 1.h.s. as $\partial F / \partial t=\left\langle\partial_{t} \mu, \delta F / \delta \mu\right\rangle$ and expand the r.h.s. as

$$
\{F, G\}(\mu)=\left\langle\mu,\left[\frac{\partial F}{\partial \mu}, \frac{\partial H}{\partial \mu}\right]\right\rangle=-\left\langle\mu, \operatorname{ad}_{\delta H / \delta \mu} \frac{\delta F}{\delta \mu}\right\rangle=\left\langle\operatorname{ad}_{\delta H / \delta \mu}^{*} \mu, \frac{\delta F}{\delta \mu}\right\rangle .
$$

Comparing these expressions gives

$$
\frac{\partial \mu}{\partial t}=\operatorname{ad}_{\delta H / \delta \mu}^{*} \mu
$$

Equations (2.8) and (2.13) define a Hamiltonian flow on $\mathfrak{v i r}^{*}$ for each choice of $H$. According to (2.8), the central charge is invariant under Hamiltonian flows; hence, the one-form $u(\theta) d \theta$ evolves according to

$$
\frac{\partial u}{\partial t}=-u^{\prime} f-2 u f^{\prime}+\frac{c}{24 \pi} f^{\prime \prime \prime}
$$

where (abusing notation slightly) $f \equiv \delta H / \delta u$. In applications, one often "works backwards:" given an equation of motion of the form (2.14), one finds a Hamiltonian for which the equation can be realized as Hamiltonian flow on $\mathfrak{v i r}^{*}$. For example, the Korteweg-de Vries $(\mathrm{KdV})$ equation was discovered long ago as a model for one-dimensional fluid flow and only much later realized as a Hamiltonian flow on $\mathfrak{v i r}^{*}$ [25]. In the next section, we will find equations governing the evolution of the boundary of three-dimensional asymptotically (A)dS spacetimes. We will realize the boundary dynamics as Hamiltonian flow on vir* by comparing the boundary's equations of motion with (2.14) and choosing $H$ appropriately.

\section{Anti-de Sitter}

The metric of three-dimensional anti-de Sitter $\left(\mathrm{AdS}_{3}\right)$ spacetime is

$$
d s^{2}=\frac{\ell^{2}}{r^{2}} d r^{2}-r^{2} d x^{+} d x^{-}
$$


where $x^{ \pm}=t / \ell \pm \phi$ are null coordinates. To study fluctuations about (3.1), we introduce a family of metrics which approaches (3.1) asymptotically, as $r \rightarrow \infty$. A seemingly reasonable ansatz for this family of metrics is

$$
d s^{2}=\frac{\ell^{2}}{r^{2}} d r^{2}-e^{2 \varphi} r^{2} d x^{+} d x^{-}+e^{4 \varphi} \gamma_{++}\left(d x^{+}\right)^{2}+e^{4 \varphi} \gamma_{--}\left(d x^{-}\right)^{2}+(\ldots),
$$

where $\varphi\left(x^{+}, x^{-}\right), \gamma_{++}\left(x^{+}, x^{-}\right)$, and $\gamma_{--}\left(x^{+}, x^{-}\right)$are independent of $r$ and $(\ldots)$ indicates subleading terms in $r^{-1}$. This ansatz is not quite right because the curvature of (3.2) falls off too slowly ${ }^{3}$ as $r \rightarrow \infty$. To fix this, take instead

$$
d s^{2}=\frac{\ell^{2}}{r^{2}} d r^{2}-\left(e^{2 \varphi} r^{2}-2 \gamma_{+-}\right) d x^{+} d x^{-}+e^{4 \varphi} \gamma_{++}\left(d x^{+}\right)^{2}+e^{4 \varphi} \gamma_{--}\left(d x^{-}\right)^{2}+(\ldots),
$$

where $\gamma_{+-}=\ell^{2} \partial_{+} \partial_{-} \varphi$. Equation (3.3) is known to be the most general asymptotically-AdS metric in three dimensions [29].

The metric functions $\varphi, \gamma_{++}$, and $\gamma_{--}$are not entirely arbitrary. They satisfy two constraints coming from the $r \rightarrow \infty$ limit of the $r \pm$-components of Einstein's equation. Following the membrane paradigm, we regard these constraints as equations of motion for a dynamical system in $1+1$ dimensions. Comparing the constraints with (2.14) will allow us to realize this 1+1-dimensional dynamics as a Hamiltonian system on a coadjoint orbit of $\mathfrak{v i r}^{*} \times \mathfrak{v i r}^{*}$ with $c=3 \ell / 2 G$.

\subsection{Boundary dynamics}

We fix a cutoff surface at large but finite $r=r_{c}$; this will serve as a proxy for the boundary of spacetime. Ultimately we are interested in the $r_{c} \rightarrow \infty$ limit. Let $n \equiv(r / \ell) \partial_{r}$ be the unit normal and $h_{\mu \nu}=g_{\mu \nu}-n_{\mu} n_{\nu}$ be the induced metric on the cutoff surface. At the cutoff, the Einstein equations enforce the constraints ${ }^{4}$

$$
\begin{aligned}
& \frac{1}{8 \pi G} \sqrt{-h} G_{n+}=\frac{\partial}{\partial x^{-}}\left[\frac{e^{4 \varphi} \gamma_{++}}{8 \pi G \ell}-\frac{\ell}{8 \pi G} \partial_{+}^{2} \varphi+\frac{\ell}{8 \pi G}\left(\partial_{+} \varphi\right)^{2}\right]=0 \\
& \frac{1}{8 \pi G} \sqrt{-h} G_{n-}=\frac{\partial}{\partial x^{+}}\left[\frac{e^{4 \varphi} \gamma_{--}}{8 \pi G \ell}-\frac{\ell}{8 \pi G} \partial_{-}^{2} \varphi+\frac{\ell}{8 \pi G}\left(\partial_{-} \varphi\right)^{2}\right]=0
\end{aligned}
$$

where terms subleading in $1 / r$ have been dropped. In section 3.3 , we will explain the fluid interpretation of (3.4)-(3.5), following the membrane paradigm. The goal for the remainder of this section is to realize these equations as a Hamiltonian flow on $\mathfrak{v i r}^{*} \times \mathfrak{v i r}^{*}$.

\footnotetext{
${ }^{3}$ Let $h_{\mu \nu}$ be the induced metric on the boundary (see below for formula). The r.h.s. of Einstein's equation gives $8 \pi G \sqrt{-h} T_{r}^{r}=O(1)$ as $r \rightarrow \infty$, violating the requirement that the metric approaches vacuum $\mathrm{AdS}_{3}$ asymptotically.

${ }^{4}$ If one sets $\gamma_{ \pm \pm}=0$, then the constraint may be interpreted as equations of motion for $\varphi$ in a flat $1+1$ spacetime. The dynamics of $\varphi$ is governed by Liouville theory [30, 31]. If instead, we consider a non-zero $\gamma_{ \pm \pm}$, as in the present case and, for example, in [6] then the boundary is curved and Liouville theory lives in a curved $1+1$ spacetime. Moreover, the \pm \pm components of the Brown-York stress-tensor are proportional to the Liouville stress-tensor while the +- components are proportional to the curvature of the boundary [32].
} 
The last two terms on the rhs's of (3.4)-(3.5) are reminiscent of the Schwarzian derivatives in the transformation law (2.9) for Virasoro coadjoint vectors. To make this correspondence precise, let $\partial_{+} \eta_{+}=e^{2 \varphi}$ and $\partial_{-} \eta_{-}=e^{2 \varphi}$. The Schwarzian derivative of $\eta_{+}$is

$$
\operatorname{Sch}\left(\eta_{+}\right)=\frac{\left(\partial_{+} \eta_{+}\right)\left(\partial_{+}^{3} \eta_{+}\right)-\frac{3}{2}\left(\partial_{+}^{2} \eta_{+}\right)^{2}}{\left(\partial_{+} \eta_{+}\right)^{2}}=-2 \partial_{+}^{2} \varphi+2\left(\partial_{+} \varphi\right)^{2} .
$$

A similar equation holds for $\operatorname{Sch}\left(\eta_{-}\right)$. Note that we always take "spatial derivatives" of $\eta_{+}$with respect to $x^{+}$and "spatial derivatives" of $\eta_{-}$with respect to $x^{-}$. The constraint equations (3.4)-(3.5) can be written compactly as

$$
\frac{\partial}{\partial x^{\mp}}\left(\Gamma_{ \pm \pm} \cdot\left(\partial_{ \pm} \eta_{ \pm}\right)^{2}-\frac{c}{24 \pi} \operatorname{Sch}\left(\eta_{ \pm}\right)\right)=0,
$$

where $\Gamma_{ \pm \pm} \equiv \gamma_{ \pm \pm} /(8 \pi G \ell)$ and $c=3 \ell / 2 G$. Comparing with (2.9), we see that $\Gamma_{++}$and $\Gamma_{--}$transform as Virasoro coadjoint vectors with identical central charges, $c=3 \ell / 2 G$. The constraint equations become

$$
\frac{\partial}{\partial x^{\mp}} \operatorname{Ad}_{\eta_{ \pm}^{-1}}^{*}\left(\Gamma_{ \pm \pm}\left(d x^{ \pm}\right)^{2}, i c\right)=0 .
$$

Using the "product rule" for the coadjoint action, ${ }^{5}$ this becomes

$$
\frac{\partial}{\partial x^{\mp}}\left(\Gamma_{ \pm \pm}\left(d x^{ \pm}\right)^{2}, i c\right)-\operatorname{ad}_{f_{ \pm}}^{*}\left(\Gamma_{ \pm \pm}\left(d x^{ \pm}\right)^{2}, i c\right)=0,
$$

where $f_{ \pm} \equiv \partial_{\mp} \eta_{ \pm} \in \mathfrak{g}$ are the adjoint vectors corresponding to the diffeomorphisms $\eta_{ \pm}$. They can be thought of as the generators of an infinite dimensional generalization of translations along $x_{ \pm}$. From this, one can read off the equation of motion for $\Gamma_{++}$which is

$$
\frac{\partial \Gamma_{++}}{\partial x^{-}}=-\left(\partial_{+} \Gamma_{++}\right) f_{+}-2 \Gamma_{++}\left(\partial_{+} f_{+}\right)+\frac{c}{24 \pi} \partial_{+}^{3} f_{+} .
$$

Notice that the dynamics of $\Gamma_{++}$is precisely of the form (2.14), with $x^{-}$playing the role of "time" and $x^{+}$playing the role of "space." So the dynamics of $\Gamma_{++}$can be realized as a Hamiltonian flow on a coadjoint orbit of $\mathfrak{v i r}^{*}$ with central charge $c=3 \ell / 2 G$; all that remains is to specify a Hamiltonian, $H_{+}$, with $\delta H_{+} / \delta \Gamma_{++}=f_{+}=\partial_{-} \eta_{+}$. This can be achieved by defining

$$
H_{+} \equiv \int_{S^{1}} f_{+} \Gamma_{++} d x^{+} .
$$

In the same way, we may regard the dynamics of $\Gamma_{--}$as a Hamiltonian flow on a second copy of $\mathfrak{v i r}^{*}$, albeit with the roles of $x^{+}$and $x^{-}$interchanged. Since the dynamics of $\Gamma_{++}$and $\Gamma_{--}$are decoupled, one can slightly abuse notation and consider a Hamiltonian, $H: \mathfrak{v i r}^{*} \times \mathfrak{v i r}^{*} \rightarrow \mathbb{R}$, for the combined system given by $H=H_{+}+H_{-}$where the first term evolves in "time" $x^{-}$and the second one in "time" $x^{+}$.

At this point, we have constructed a Hamiltonian flow on $\mathfrak{v i r}^{*} \times \mathfrak{v i r}^{*}$ which may be studied as an interesting dynamical system in its own right, independent of its origins in $\mathrm{AdS}_{3}$ gravity. The equation of motion (3.10) is reminiscent of the Korteweg-de Vries equation, another example of a Hamiltonian flow on $\mathfrak{v i r}^{*}$ [25]. The unusual feature of our Hamiltonian is the arbitrary function, $f_{ \pm}\left(x^{+}, x^{-}\right)$. In some sense, we really have a family of Hamiltonian systems parametrized by $f_{ \pm}\left(x^{+}, x^{-}\right)$.

\footnotetext{
${ }^{5}$ See Proposition 9.3 .8 of [3].
} 


\subsection{Charge algebra}

Returning to the boundary equations of motion (3.8), we have the conservation law

$$
\frac{\partial}{\partial x^{-}} \Xi_{++}\left(x^{+}, x^{-}\right)=0,
$$

where $\Xi_{++} \equiv \operatorname{Ad}_{\eta_{+}^{-1}}^{*}\left(\Gamma_{++}\left(d x^{+}\right)^{2}, i c\right)$ is an element of $\mathfrak{v i r}^{*}$ and we regard $x^{-}$as time. Now, $\mathfrak{v i r}^{*}$ is an infinite dimensional vector space, so projecting (3.12) onto a basis for vir gives an infinite set of conserved charges. The usual choice is

$$
v_{m}^{+}=\left(e^{i m x^{+}} \partial_{+},-\frac{i}{24} \delta_{m}^{0}\right) .
$$

Projecting onto this basis using (2.6) gives an infinite set of conserved charges,

$$
\mathcal{Q}_{m}^{+} \equiv\left\langle\Xi_{++}\left(x^{+}\right), v_{m}^{+}\left(x^{+}\right)\right\rangle=\text {const. }
$$

The Lie-Poisson bracket (2.10) of the charges is

$$
\begin{aligned}
i\left\{\mathcal{Q}_{m}^{+}, \mathcal{Q}_{n}^{+}\right\}\left(\Xi_{++}\right) & =\left\langle\Xi_{++}, i\left[v_{m}^{+}, v_{n}^{+}\right]\right\rangle \\
& =(m-n)\left\langle\Xi_{++}, v_{m+n}\right\rangle+\frac{1}{12} m\left(m^{2}-1\right) \delta_{m+n}^{0}\langle(0, i c),(0,-i)\rangle \\
& =(m-n) \mathcal{Q}_{m+n}^{+}+\frac{c}{12} m\left(m^{2}-1\right) \delta_{m+n}^{0}
\end{aligned}
$$

We get a second copy of this algebra by considering the boundary equation of motion $\partial_{+} \Xi_{--}=0$ and regarding $x^{+}$as "time." In this case, the Lie-Poisson brackets of the corresponding charges, say $\mathcal{Q}_{m}^{-}$, are

$$
\begin{aligned}
i\left\{\mathcal{Q}_{m}^{-}, \mathcal{Q}_{n}^{-}\right\} & =(m-n) \mathcal{Q}_{m+n}^{-}+\frac{c}{12} m\left(m^{2}-1\right) \delta_{m+n}^{0}, \\
i\left\{\mathcal{Q}_{m}^{+}, \mathcal{Q}_{n}^{-}\right\} & =0
\end{aligned}
$$

This is the asymptotic charge algebra of $\mathrm{AdS}_{3}$ gravity derived long ago by Brown and Henneaux [4], with central charge $c=3 \ell / 2 G$. We have arrived at this result in a new way: by regarding the constraints on the boundary data as a 1+1-dimensional Hamiltonian system and using the corresponding Lie-Poisson bracket (2.10) on $\mathfrak{v i r}^{*} \times \mathfrak{v i r}{ }^{*}$ to compute the charge algebra. The simplicity of the Lie-Poisson bracket on $\mathfrak{v i r}^{*} \times \mathfrak{v i r}^{*}$ has helped to streamline the derivation.

\subsection{Membrane paradigm}

The idea of regarding the constraints on the boundary data as a dynamical system in $1+1$ dimensions is inspired by the black hole membrane paradigm. In this section, we will use the relationship with the membrane paradigm to clarify the connection between gravity and fluid dynamics.

Previously we introduced a cutoff surface at large but finite $r=r_{c}$, with induced metric $h_{\mu \nu}$ and unit normal $n$. This is the analogue of the "stretched horizon" of the black hole membrane paradigm. Following the membrane paradigm, we now assign the cutoff surface 
a stress-energy tensor, $t_{\mu \nu}$. The stress-energy tensor is defined such that it terminates the gravitational field at the cutoff. The Israel junction condition gives

$$
t_{\mu \nu}=-\frac{1}{8 \pi G}\left(K_{\mu \nu}-\left(K-\frac{1}{\ell}\right) h_{\mu \nu}\right)
$$

where $K_{\nu}^{\mu}=h^{\delta}{ }_{\nu} \nabla_{\delta} n^{\mu}$ is the extrinsic curvature of the cutoff surface and $K$ is its trace. The term proportional to $1 / \ell$ is a regularization term added in order to have a finite stress tensor as we approach the boundary ${ }^{6}[33]$. Plugging in the metric (3.3) gives

$$
t_{ \pm \pm}=\frac{e^{4 \varphi} \gamma_{ \pm \pm}}{8 \pi G \ell}=e^{4 \varphi} \Gamma_{ \pm \pm}
$$

for the diagonal components, while the off-diagonal ones are proportional to the Ricci scalar of the cutoff surface. Now, the variables $\Gamma_{ \pm \pm}$introduced earlier may be interpreted as energy densities. They are the energy densities of the boundary stress-energy tensor as viewed by null observers with four-momenta $k_{ \pm}=e^{-2 \varphi} \partial_{ \pm}$. It is interesting to look at the trace of the stress-energy tensor where we already see a hint of conformal invariance [33]:

$$
t^{\mathrm{reg}}=\frac{c}{24 \pi} R_{2 d}, \quad c=\frac{3 \ell}{2 G},
$$

where $R_{2 d}=8 e^{-2 \varphi} \gamma_{+-} /(r \ell)^{2}$ is the Ricci scalar of the cutoff surface. This corresponds to the trace anomaly of a conformal field theory with central charge ${ }^{7} c=3 \ell / 2 G$. This anomaly only exists for odd bulk dimensions. In these cases, a logarithmic divergence in the action appears and cannot be canceled by a polynomial counterterm without including a cutoff dependence [34-38]. Given that the stress tensor measures the change in the action due to perturbations of the boundary, one finds this anomaly by looking at the trace of (3.19).

Now, we move on to analyze the dynamics. Earlier we obtained equations of motion (3.4)-(3.5) for $\Gamma_{ \pm \pm}=e^{-4 \varphi} t_{ \pm \pm}$by projecting Einstein's equations onto the cutoff surface. We obtain the same conservation laws by imposing conservation of $t_{\mu \nu}$ :

$$
\sqrt{-h} h_{a \mu} t_{\mid \nu}^{\mu \nu}=0
$$

where the covariant derivative is $t^{\mu \nu}{ }_{\mid \nu}=h_{\nu}^{\delta} \nabla_{\delta} t^{\mu \nu}$ and the index $a$ corresponds to $x_{ \pm}$. This means that the constraint equations (3.4)-(3.5) may be interpreted as energy conservation laws for the boundary stress-energy tensor.

Ordinary (inviscid, nonrelativistic) fluids are governed by the compressible Euler equations. Mass conservation is described by the continuity equation

$$
\frac{\partial \rho}{\partial t}+\nabla \cdot(\rho v)=0
$$

\footnotetext{
${ }^{6}$ Note, our correction differs from [33] by a minus sign because we use a different convention for defining the extrinsic curvature.

${ }^{7}$ Note that compared to [33] we differ by a minus sign in the conformal anomaly. This is due to the fact that our conventions for the Riemann tensor differ by a minus sign.
} 
where $\rho$ is the fluid's mass density and $v$ is its velocity. Let $X$ and $x$ denote the positions of a fluid parcel at $t=0$ and time $t$, respectively. These are called Lagrangian and Eulerian coordinates. Consider the diffeomorphism $\eta: X \rightarrow x$. It is related to the fluid velocity by

$$
\frac{\partial \eta(X, t)}{\partial t}=v(\eta(X, t), t) .
$$

In these variables, the continuity equation (3.22) is

$$
\frac{\partial}{\partial t} \operatorname{Ad}_{\eta^{-1}}^{*}(\rho(x) d x)=0
$$

where $\operatorname{Ad}_{\eta^{-1}}^{*}(\rho(x) d x)=\eta^{*}(\rho(x) d x)$ is the adjoint action of $\operatorname{Diff}(M)$ on $\Omega^{n}(M)$. Here $M$ is the manifold on which the fluid is flowing and $\Omega^{n}(M)$ is the space of $n$-forms on $M$.

Recall that the dynamics governing the $\mathrm{AdS}_{3}$ boundary was described by

$$
\frac{\partial}{\partial x^{\mp}} \operatorname{Ad}_{\eta_{ \pm}^{-1}}^{*}\left(\Gamma_{ \pm \pm}\left(d x^{ \pm}\right)^{2}, i c\right)=0 .
$$

Comparing (3.24) and (3.25), we see that the maps $\eta_{ \pm}$are analogous to the map $\eta$ from Lagrangian to Eulerian coordinates. In fact, the gravity equations (3.25) are almost equivalent to two copies of the usual fluid continuity equation, with the only difference being that the dynamics takes place on $\mathfrak{v i r}^{*}$ rather than $\Omega^{n}(M)$.

\section{4 de Sitter}

The metric of three-dimensional de Sitter space $\left(\mathrm{dS}_{3}\right)$ is

$$
d s^{2}=-\frac{\ell^{2}}{r^{2}} d r^{2}+r^{2} d t^{+} d t^{-}
$$

where $t^{ \pm}=t / \ell \pm i \phi$. This is related to the $\mathrm{AdS}_{3}$ metric (3.1) by the substitution $\ell \rightarrow-i \ell$, in which case $t^{ \pm} \rightarrow i x^{ \pm}$. In this section, we extend our earlier discussion to asymptotically $\mathrm{dS}_{3}$ spacetimes. The calculations largely resemble the $\mathrm{AdS}_{3}$ case, so we will be concise.

To begin, we introduce a family of metrics which approaches (4.1) asymptotically, as $r \rightarrow \infty$ :

$$
d s^{2}=-\frac{\ell^{2}}{r^{2}} d r^{2}+\left(e^{2 \varphi} r^{2}-2 L_{+-}\right) d t^{+} d t^{-}-e^{4 \varphi} L_{++}\left(d t^{+}\right)^{2}-e^{4 \varphi} L_{--}\left(d t^{-}\right)^{2}+(\ldots),
$$

where $L_{+-} \equiv \ell^{2} \partial_{+} \partial_{-} \varphi$. This is the $\mathrm{dS}_{3}$ analogue of (3.3). The metric functions $\varphi\left(t^{+}, t^{-}\right)$, $L_{++}\left(t^{+}, t^{-}\right)$, and $L_{--}\left(t^{+}, t^{-}\right)$are independent of $r$. Note that outside the cosmological horizon, $r$ is a timelike coordinate and $t$ is a spacelike coordinate.

As before, we fix a cutoff surface at large but finite $r=r_{c}$. This is a proxy for the "boundary of spacetime" at $r \rightarrow \infty$. In the $\mathrm{AdS}_{3}$ case, the cutoff was a timelike surface but now it is spacelike. Let $n \equiv(r / \ell) \partial_{r}$ be the outward pointing unit normal and $h_{\mu \nu}=g_{\mu \nu}+n_{\mu} n_{\nu}$ be the induced metric on the cutoff surface. The Einstein equations enforce the constraints

$$
\begin{aligned}
& \frac{1}{8 \pi G} \sqrt{h} G_{n+}=\frac{\partial}{\partial t^{-}}\left[\frac{e^{4 \varphi} L_{++}}{8 \pi G \ell}-\frac{\ell}{8 \pi G} \partial_{+}^{2} \varphi+\frac{\ell}{8 \pi G}\left(\partial_{+} \varphi\right)^{2}\right]=0, \\
& \frac{1}{8 \pi G} \sqrt{h} G_{n-}=\frac{\partial}{\partial t^{+}}\left[\frac{e^{4 \varphi} L_{--}}{8 \pi G \ell}-\frac{\ell}{8 \pi G} \partial_{-}^{2} \varphi+\frac{\ell}{8 \pi G}\left(\partial_{-} \varphi\right)^{2}\right]=0 .
\end{aligned}
$$


Following the $\mathrm{AdS}_{3}$ example, we set $\mathcal{L}_{ \pm \pm} \equiv L_{ \pm \pm} /(8 \pi G \ell), \partial_{+} \eta_{+}=e^{2 \varphi}$, and $\partial_{-} \eta_{-}=e^{2 \varphi}$ (in this section, $\partial_{ \pm}=\partial_{t^{ \pm}}$). Further define

$$
\Theta_{ \pm \pm} \equiv \operatorname{Ad}_{\eta_{ \pm}^{-1}}^{*}\left(\mathcal{L}_{ \pm \pm}\left(\mathrm{d} t^{ \pm}\right)^{2}, i c\right)
$$

The constraint equations (4.3)-(4.4) become simply

$$
\partial_{\mp} \Theta_{ \pm \pm}=0 .
$$

So we are in the same situation as before. As in the $\mathrm{AdS}_{3}$ case, the constraints give a Hamiltonian system on $\mathfrak{v i r}^{*} \times \mathfrak{v i r}^{*}$ with identical central charges $c=3 \ell / 2 G$. Note that the de Sitter boundary is a spacelike surface, while the anti de Sitter boundary was a timelike surface. So one might except to see an interchange of the notions of "time" and "space" in the boundary dynamics. This is somewhat obscured by the fact that we are using null coordinates. In one sector of the de Sitter boundary dynamics, the equation of motion is $\partial_{-} \Theta_{++}=0$ and $t^{-}$is regarded as time while $t^{+}$is regarded as space. In the other sector of the de Sitter boundary dynamics, the equation of motion is $\partial_{+} \Theta_{--}=0$ and $t^{+}$is regarded as time while $t^{-}$is regarded as space. Now the AdS and dS metrics are related by the Wick rotation $\ell \rightarrow i \ell$. This sends the AdS null coordinate $x^{+}$to the dS null coordinate $t^{-}$and it sends $x^{-}$to $t^{+}$. So in that sense, passing from AdS to dS interchanges space and time.

\subsection{Conserved quantities}

The conserved quantities, $\Theta_{ \pm \pm}$, are elements of $\mathfrak{v i r}^{*} \times \mathfrak{v i r}^{*}$. As before, we may project the conserved quantities onto a basis of $\mathfrak{v i r} \times \mathfrak{v i r}$. In the present case, a natural basis for the left Virasoro is

$$
l_{m}^{+}=\left(e^{-m t_{+}} \partial_{+}, \frac{1}{24} \delta_{m}^{0}\right)
$$

and the Lie brackets are

$$
\left[l_{m}^{+}, l_{n}^{+}\right]=(m-n) l_{m+n}^{+}+\frac{1}{12}\left(m^{3}-m\right) \delta_{m+n}^{0} \mathcal{Z}^{\prime},
$$

where $\mathcal{Z}^{\prime}=(0,1)$. The conserved quantities are

$$
L_{m}^{+} \equiv\left\langle l_{m}^{+}\left(t^{+}\right),-i \Theta_{++}\left(t^{+}\right)\right\rangle=\text {const. }
$$

and the Lie-Poisson bracket is

$$
\left\{L_{m}^{+}, L_{n}^{+}\right\}\left(-i \Theta_{++}\right)=(m-n) L_{m+n}^{+}+\frac{c}{12}\left(m^{3}-m\right) \delta_{m+n}^{0} .
$$

The renormalized Brown-York stress energy tensor is now

$$
t_{\mu \nu}^{\mathrm{dS}}=-\frac{1}{8 \pi G}\left(K_{\mu \nu}-\left(K-\frac{1}{\ell}\right) h_{\mu \nu}\right),
$$

whose diagonal components are

$$
t_{ \pm \pm}^{\mathrm{dS}}=-e^{4 \varphi} \mathcal{L}_{ \pm \pm}
$$




\subsection{Example: Kerr-dS 3}

To gain some intuition into the meaning of the $L_{ \pm \pm}$we can look at an example of an asymptotically $\mathrm{dS}_{3}$ spacetime. Although there are no black hole solutions in $\mathrm{dS}_{3}$, there are conical defects like the Kerr- $\mathrm{dS}_{3}$ spacetime [39-41]. The metric for Kerr- $\mathrm{dS}_{3}$ is

$$
\begin{aligned}
d s^{2}= & -\left(8 G M-\frac{r^{2}}{\ell^{2}}+\frac{(8 G J)^{2}}{4 r^{2}}\right) d t^{2}+\left(8 G M-\frac{r^{2}}{\ell^{2}}+\frac{(8 G J)^{2}}{4 r^{2}}\right)^{-1} d r^{2} \\
& +r^{2}\left(-\frac{8 G J}{2 r^{2}} d t+d \phi\right)^{2} .
\end{aligned}
$$

It is a quotient of $\mathrm{dS}_{3}$ by a discrete group [38] similar to the BTZ black hole [42]. Rewriting this metric in $t_{ \pm}$coordinates and taking $t_{ \pm} \rightarrow\left(1+2 G M \ell^{2} / r^{2}\right) t_{ \pm}$we find that at large $r$,

$$
d s^{2}=-\frac{\ell^{2}}{r^{2}} d r^{2}+r^{2} d t^{+} d t^{-}-2 G \ell(\ell M+i J)\left(d t^{+}\right)^{2}-2 G \ell(\ell M-i J)\left(d t^{-}\right)^{2}+(\ldots) .
$$

Comparing with (4.2), we find that the mass and angular momentum are

$$
M=\frac{L_{++}+L_{--}}{4 G \ell^{2}}, \quad J=i \frac{L_{++}-L_{--}}{4 G \ell} .
$$

Note that the $L_{ \pm \pm}$are complex valued.

\section{Discussion}

We analyzed the dynamics of the asymptotic data of $\mathrm{dS}_{3}$ and $\mathrm{AdS}_{3}$ by realizing Einstein's equations as a 1+1-dimensional Hamiltonian system. We constructed an infinite set of conserved charges by projecting the conservation laws onto a basis for vir and we computed the asymptotic charge algebra using the Lie-Poisson bracket. The Hamiltonian flow on $\mathfrak{g}^{*}$ is restricted to coadjoint orbits (cf. eq. (3.9)), which are symplectic manifolds, so they are natural phase spaces for classical dynamics. Moreover, coadjoint orbits of $\mathfrak{g}^{*}$ can be identified with representations of the group $G$ according to Kirillov's orbit method [26]. So this formulation of the classical theory can give insights into its quantization, see [43-49] for some approaches.

As we mentioned at the outset, the subject of asymptotic symmetries has a wide range of applications, and our results give a new perspective on these applications. For example, in cosmology, the change in the curvature perturbation, $\zeta$, produced by an asymptotic symmetry transformation of $\mathrm{dS}_{3}$ is identified with adiabatic modes of $\zeta$ [50]. The relation to the membrane paradigm described in the present paper allows one to interpret the perturbations produced by adiabatic modes as changes in the energy density of the boundary fluid as observed by a null observer (cf. eq. (4.12)). Mathematically, they are paths along coadjoint orbits of $\mathfrak{v i r}^{*} \times \mathfrak{v i r}^{*}$. It would be interesting to understand the relationship between our formalism and [51], which outlines a connection between microstates of the membrane at the horizon and Bekenstein-Hawking entropy. 


\section{Acknowledgments}

It is a pleasure to thank Justin Khoury for helpful discussions. MCG is supported in part by US Department of Energy (HEP) Award de-sc0013528. RFP is supported by a Prize Postdoctoral Fellowship in the Natural Sciences at Columbia University and by Simons Foundation Award Number 555117.

Open Access. This article is distributed under the terms of the Creative Commons Attribution License (CC-BY 4.0), which permits any use, distribution and reproduction in any medium, provided the original author(s) and source are credited.

\section{References}

[1] R.M. Wald and A. Zoupas, A General definition of 'conserved quantities' in general relativity and other theories of gravity, Phys. Rev. D 61 (2000) 084027 [gr-qc/9911095] [INSPIRE].

[2] G. Barnich and F. Brandt, Covariant theory of asymptotic symmetries, conservation laws and central charges, Nucl. Phys. B 633 (2002) 3 [hep-th/0111246] [INSPIRE].

[3] J. Marsden and T. Ratiu, Introduction to Mechanics and Symmetry: A Basic Exposition of Classical Mechanical Systems, Texts in Applied Mathematics, Springer New York, (2002).

[4] J.D. Brown and M. Henneaux, Central Charges in the Canonical Realization of Asymptotic Symmetries: An Example from Three-Dimensional Gravity, Commun. Math. Phys. 104 (1986) 207 [INSPIRE].

[5] G. Compère, W. Song and A. Strominger, New Boundary Conditions for AdS3, JHEP 05 (2013) 152 [arXiv: 1303.2662] [INSPIRE].

[6] C. Troessaert, Enhanced asymptotic symmetry algebra of AdS $S_{3}$, JHEP 08 (2013) 044 [arXiv: 1303.3296] [INSPIRE].

[7] A. Pérez, D. Tempo and R. Troncoso, Boundary conditions for General Relativity on AdS $S_{3}$ and the KdV hierarchy, JHEP 06 (2016) 103 [arXiv: 1605.04490] [INSPIRE].

[8] H.A. González, J. Matulich, M. Pino and R. Troncoso, Revisiting the asymptotic dynamics of General Relativity on $A d S_{3}$, JHEP 12 (2018) 115 [arXiv: 1809.02749] [InSPIRE].

[9] K.S. Thorne, R.H. Price and D.A. MacDonald, Black Holes: The Membrane Paradigm, Yale University Press (1986) [INSPIRE].

[10] M. Parikh and F. Wilczek, An Action for black hole membranes, Phys. Rev. D 58 (1998) 064011 [gr-qc/9712077] [INSPIRE].

[11] R.F. Penna, Energy extraction from boosted black holes: Penrose process, jets and the membrane at infinity, Phys. Rev. D 91 (2015) 084044 [arXiv:1503.00728] [InSPIRE].

[12] J.E. Marsden, T. Ratiu and A. Weinstein, Semidirect products and reduction in mechanics, Trans. Am. Math. Soc. 281 (1984) 147.

[13] R.F. Penna, BMS invariance and the membrane paradigm, JHEP 03 (2016) 023 [arXiv:1508.06577] [INSPIRE].

[14] C. Eling and Y. Oz, On the Membrane Paradigm and Spontaneous Breaking of Horizon BMS Symmetries, JHEP 07 (2016) 065 [arXiv: 1605.00183] [INSPIRE]. 
[15] C. Eling, Spontaneously Broken Asymptotic Symmetries and an Effective Action for Horizon Dynamics, JHEP 02 (2017) 052 [arXiv: 1611.10214] [INSPIRE].

[16] R.F. Penna, Near-horizon BMS symmetries as fluid symmetries, JHEP 10 (2017) 049 [arXiv: 1703.07382] [INSPIRE].

[17] G. Barnich and B. Oblak, Holographic positive energy theorems in three-dimensional gravity, Class. Quant. Grav. 31 (2014) 152001 [arXiv: 1403.3835] [INSPIRE].

[18] G. Barnich and B. Oblak, Notes on the BMS group in three dimensions: II. Coadjoint representation, JHEP 03 (2015) 033 [arXiv: 1502.00010] [INSPIRE].

[19] B. Oblak, BMS Particles in Three Dimensions, Ph.D. Thesis, Brussels U. (2016) [DOI:10.1007/978-3-319-61878-4] [arXiv: 1610.08526] [INSPIRE].

[20] R.F. Penna, $B M S_{3}$ invariant fluid dynamics at null infinity, Class. Quant. Grav. 35 (2018) 044002 [arXiv: 1708.08470] [INSPIRE].

[21] J.M. Maldacena, Non-Gaussian features of primordial fluctuations in single field inflationary models, JHEP 05 (2003) 013 [astro-ph/0210603] [INSPIRE].

[22] K. Hinterbichler, L. Hui and J. Khoury, An Infinite Set of Ward Identities for Adiabatic Modes in Cosmology, JCAP 01 (2014) 039 [arXiv:1304.5527] [INSPIRE].

[23] L. Berezhiani and J. Khoury, Slavnov-Taylor Identities for Primordial Perturbations, JCAP 02 (2014) 003 [arXiv: 1309.4461] [INSPIRE].

[24] A. Strominger, Lectures on the Infrared Structure of Gravity and Gauge Theory, arXiv: 1703.05448 [INSPIRE].

[25] B. Khesin and R. Wendt, The Geometry of Infinite-Dimensional Groups, A series of modern surveys in mathematics, Springer Berlin Heidelberg (2008).

[26] A.A. Kirillov, Lectures on the Orbit Method, vol. 64, American Mathematical Society, Providence, RI (2004).

[27] C. Bunster and A. Pérez, Superselection rule for the cosmological constant in three-dimensional spacetime, Phys. Rev. D 91 (2015) 024029 [arXiv:1412.1492] [InSPIRE].

[28] S. Weinberg, The Quantum theory of fields. Vol. 1: Foundations, Cambridge University Press (2005) [INSPIRE].

[29] G. Barnich and C. Troessaert, Aspects of the BMS/CFT correspondence, JHEP 05 (2010) 062 [arXiv: 1001.1541 ] [INSPIRE].

[30] O. Coussaert, M. Henneaux and P. van Driel, The Asymptotic dynamics of three-dimensional Einstein gravity with a negative cosmological constant, Class. Quant. Grav. 12 (1995) 2961 [gr-qc/9506019] [INSPIRE].

[31] S. Cacciatori and D. Klemm, The Asymptotic dynamics of de Sitter gravity in three-dimensions, Class. Quant. Grav. 19 (2002) 579 [hep-th/0110031] [INSPIRE].

[32] V. Balasubramanian, J. de Boer and D. Minic, Notes on de Sitter space and holography, Class. Quant. Grav. 19 (2002) 5655 [hep-th/0207245] [INSPIRE].

[33] V. Balasubramanian and P. Kraus, A Stress tensor for Anti-de Sitter gravity, Commun. Math. Phys. 208 (1999) 413 [hep-th/9902121] [INSPIRE].

[34] M. Henningson and K. Skenderis, The Holographic Weyl anomaly, JHEP 07 (1998) 023 [hep-th/9806087] [INSPIRE]. 
[35] R. Emparan, C.V. Johnson and R.C. Myers, Surface terms as counterterms in the AdS/CFT correspondence, Phys. Rev. D 60 (1999) 104001 [hep-th/9903238] [INSPIRE].

[36] S. de Haro, S.N. Solodukhin and K. Skenderis, Holographic reconstruction of space-time and renormalization in the AdS/CFT correspondence, Commun. Math. Phys. 217 (2001) 595 [hep-th/0002230] [INSPIRE].

[37] K. Skenderis, Asymptotically Anti-de Sitter space-times and their stress energy tensor, Int. J. Mod. Phys. A 16 (2001) 740 [hep-th/0010138] [InSPIRE].

[38] V. Balasubramanian, J. de Boer and D. Minic, Mass, entropy and holography in asymptotically de Sitter spaces, Phys. Rev. D 65 (2002) 123508 [hep-th/0110108] [InSPIRE].

[39] S. Deser and R. Jackiw, Three-Dimensional Cosmological Gravity: Dynamics of Constant Curvature, Annals Phys. 153 (1984) 405 [INSPIRE].

[40] M.-I. Park, Statistical entropy of three-dimensional Kerr-de Sitter space, Phys. Lett. B 440 (1998) 275 [hep-th/9806119] [INSPIRE].

[41] M. Bañados, T. Brotz and M.E. Ortiz, Quantum three-dimensional de Sitter space, Phys. Rev. D 59 (1999) 046002 [hep-th/9807216] [INSPIRE].

[42] M. Bañados, C. Teitelboim and J. Zanelli, The Black hole in three-dimensional space-time, Phys. Rev. Lett. 69 (1992) 1849 [hep-th/9204099] [INSPIRE].

[43] E. Witten, Coadjoint Orbits of the Virasoro Group, Commun. Math. Phys. 114 (1988) 1 [INSPIRE].

[44] A. Alekseev and S.L. Shatashvili, Path Integral Quantization of the Coadjoint Orbits of the Virasoro Group and 2D Gravity, Nucl. Phys. B 323 (1989) 719 [InSPIRE].

[45] A. Alekseev, L.D. Faddeev and S.L. Shatashvili, Quantization of symplectic orbits of compact Lie groups by means of the functional integral, J. Geom. Phys. 5 (1988) 391 [InSPIRE].

[46] H.L. Verlinde and E.P. Verlinde, Conformal field theory and geometric quantization, in Trieste School and Workshop on Superstrings, Trieste, Italy, April 3-14, 1989, pp. 422-449 (1989) [INSPIRE].

[47] A. Alekseev and S.L. Shatashvili, From geometric quantization to conformal field theory, Commun. Math. Phys. 128 (1990) 197 [INSPIRE].

[48] A. Alekseev and S.L. Shatashvili, Coadjoint Orbits, Cocycles and Gravitational Wess-Zumino, arXiv:1801.07963 [INSPIRE].

[49] J. Cotler and K. Jensen, A theory of reparameterizations for AdS $S_{3}$ gravity, JHEP 02 (2019) 079 [arXiv: 1808.03263] [INSPIRE].

[50] K. Hinterbichler, A. Joyce and J. Khoury, Inflation in Flatland, JCAP 01 (2017) 044 [arXiv: 1609.09497] [INSPIRE].

[51] D. Grumiller and M.M. Sheikh-Jabbari, Membrane Paradigm from Near Horizon Soft Hair, Int. J. Mod. Phys. D 27 (2018) 1847006 [arXiv:1805.11099] [INSPIRE]. 